\title{
TRASTORNO BIPOLAR EN EL EMBARAZO: TRATAR O NO TRATAR
}

\section{BIPOLAR DISORDER IN PREGNANCY: TO TREAT OR NOT TO TREAT}

\section{Raúl REAL ${ }^{1}$.}

${ }^{1}$ Profesor Adjunto de Medicina Interna, Facultad de Ciencias Médicas, Universidad Nacional de Asunción, San Lorenzo - Paraguay. Miembro del Comité Editorial de la Revista Medicina Clínica y Social.

Cómo citar este artículo: Real R. Trastorno bipolar en el embarazo: tratar o no tratar. Medicina Clínica y Social. 2017;1(2):184-185.

\section{Estimado Editor,}

El trastorno bipolar es una afección de creciente incidencia (1). Los factores de riesgo son diversos, pero los recientemente identificados son el síndrome del intestino irritable y las adversidades en la infancia, mientras que la obesidad, el asma, la seropositividad para Toxoplasma gondii y traumatismo craneano han demostrado escasa relación (2).

Las mujeres en fase perinatal con trastorno bipolar se caracterizan por escasos síntomas residuales de depresión independientemente de la farmacoterapia lo que lo que supone un riesgo de recidiva y de deterioro de la crianza de los hijos. Por ello, el tratamiento de las mujeres embarazadas con este trastorno merece atención del personal de salud y la investigación para mejorar los resultados psiquiátricos (3).

Un reciente estudio ha confirmado el efecto teratogénico del litio, sobre todo en la aparición de la malformación de Ebstein, aunque se pudo comprobar que el efecto no es tan considerable en comparación con la lamotrigina (4).

El artículo publicado por Rodríguez $\mathrm{H}$ en el volumen 1 número 1 de Medicina Clínica y Social plantea el dilema de continuar o suspender el tratamiento de la gestante con trastorno bipolar sabiendo que ambas decisiones tiene sus riesgos, sobre todo a nivel perinatal (5). Lamentablemente no existe aún un consenso o guía bien establecida para esta disyuntiva (6).

La inclusión de mujeres en ensayos clínicos es necesaria para obtener resultados válidos pues la generalización de estudios en varones puede conducir a conclusiones erróneas debido a las diferencias biológicas entre los géneros (7).

El artículo de Rodríguez $\mathrm{H}$ expresa claramente que es necesario un consentimiento informado para el tratamiento del trastorno bipolar en las gestantes. Como médicos generales o especialistas, no debemos dejar de usar esa herramienta y consultar a los Comités de Ética institucional para tomar estas decisiones.

\section{CONFLICTOS DE INTERÉS Y FUENTE DE FINANCIACIÓN}

El autor declara no poseer conflictos de interés. Fuente de financiación: ninguna. 


\section{REFERENCIAS BIBLIOGRÁFICAS}

1. Post RM, Altshuler LL, Kupka R, McElroy SL, Frye MA, Rowe M, et al. More childhood onset bipolar disorder in the United States than Canada or Europe: Implications for treatment and prevention. Neurosci Biobehav Rev. 2017;74(Pt A):204-213. https://doi.org/10.1016/j.neubiorev.2017.01.022

2. Bortolato B, Köhler CA, Evangelou E, León-Caballero J, Solmi M, Stubbs B, et al. Systematic assessment of environmental risk factors for bipolar disorder: an umbrella review of systematic reviews and meta-analyses. Bipolar Disord. 2017;19(2):84-96. https://doi.org/10.1111/bdi.12490

3. Driscoll KE, Sit DKY, Moses-Kolko EL, Pinheiro E, Yang A, Ciolino JD, et al. Mood symptoms in pregnant and postpartum women with bipolar disorder: a naturalistic study. Bipolar Disord. 2017;19(4):295-304. https://doi.org/10.1111/bdi.12500

4. Patorno E, Huybrechts KF, Bateman BT, Cohen JM, Desai RJ, Mogun H, et al. Lithium Use in Pregnancy and the Risk of Cardiac Malformations. N Engl J Med. 2017;376(23):2245-2254. https://doi.org/10.1056/NEJMoa1612222

5. Rodríguez $\mathrm{H}$. Tratamiento farmacológico del trastorno bipolar en el embarazo. Medicina Clínica y Social. 2017;1(1):42-48. URL.

6. Broeks SC, Thisted Horsdal H, Glejsted Ingstrup K, Gasse C. Psychopharmacological drug utilization patterns in pregnant women with bipolar disorder - A nationwide register-based study. J Affect Disord. 2017;210:158165. https://doi.org/10.1016/j.jad.2016.12.001

7. Committee Opinion No. 646 Summary: Ethical Considerations for Including Women as Research Participants. Obstet Gynecol. 2015;126(5):e100-107. https://doi.org/10.1097/AOG.0000000000001150 